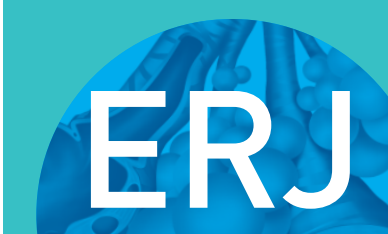

open research

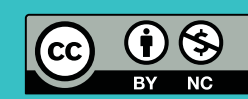

\section{The inflammasome pathway in stable COPD and acute exacerbations}

\author{
Rosa Faner ${ }^{1,2,9}$, Patricia Sobradillo ${ }^{2,3,9}$, Aina Noguera ${ }^{2,4}$, Cristina Gomez ${ }^{4}$, \\ Tamara Cruz ${ }^{1,2}$, Alejandra López-Giraldo ${ }^{1,2}$, Eugeni Ballester ${ }^{5,6}$, Nestor Soler ${ }^{5,6}$, \\ Juan I. Arostegui6,7, Pablo Pelegrín ${ }^{8}$, Roberto Rodriguez-Roisin ${ }^{2,5,6}$, \\ Jordi Yagüe $e^{6,7}$, Borja G. Cosio ${ }^{2,4}$, Manel Juan $^{6,7}$ and Alvar Agustí $i^{1,2,5,6}$
}

Affiliations: ${ }^{1}$ Fundació Clinic per a la Recerca Biomèdica, Barcelona, Spain. ${ }^{2}$ CIBER Respiratory Diseases (CIBERES), Barcelona, Spain. ${ }^{3}$ Pulmonary Service, Hospital Txagorritxu, Vitoria, Spain. ${ }^{4}$ University Hospital Son Espases-IdISPa, Palma de Mallorca, Spain. ${ }^{5}$ Institut Respiratori, Hospital Clinic, Barcelona, Spain. ${ }^{6}$ Biomedic Research Institute August Pi i Sunyer (IDIBAPS), Barcelona, Spain. ${ }^{7}$ Immunology Dept, Hospital Clinic, Barcelona, Spain. ${ }^{8}$ Inflammation and Experimental Surgery Unit, CIBERHED, Murcia's BioHealth, Research Institute IMIB-Arrixaca, University Hospital Virgen de la Arrixaca, Murcia, Spain. ${ }^{9}$ These authors contributed equally to this work.

Correspondence: Alvar Agustí, Respiratory Institute, Hospital Clinic, Villarroel 170 (Escalera 3, Planta 5), Barcelona 08036, Spain. E-mail: alvar.agustidaclinic.ub.es

ABSTRACT Chronic obstructive pulmonary disease (COPD) is characterised by pulmonary and systemic inflammation that bursts during exacerbations of the disease (ECOPD). The NLRP3 inflammasome is a key regulatory molecule of the inflammatory response. Its role in COPD is unclear.

We investigated the NLRP3 inflammasome status in: 1) lung tissue samples from 38 patients with stable COPD, 15 smokers with normal spirometry and 14 never-smokers; and 2) sputum and plasma samples from 56 ECOPD patients, of whom 41 could be reassessed at clinical recovery.

We observed that: 1) in lung tissue samples of stable COPD patients, NLRP3 and interleukin (IL)-1 $\beta$ mRNA were upregulated, but both caspase-1 and ASC were mostly in inactive form, and 2) during infectious ECOPD, caspase-1, oligomeric ASC and associated cytokines (IL-1 $\beta$, IL-18) were significantly increased in sputum compared with clinical recovery.

The NLRP3 inflammasome is primed, but not activated, in the lungs of clinically stable COPD patients. Inflammasome activation occurs during infectious ECOPD. The results of this study suggest that the inflammasome participates in the inflammatory burst of infectious ECOPD.

@ERSpublications

The NLRP3 inflammasome is primed in stable COPD lungs, then activated during infectious exacerbation http://ow.ly/Wopi300DXcT

This article has supplementary material available from openres.ersjournals.com

Received: Jan 022016 | Accepted after revision: May 172016

Support statement: This study was supported, in part, by Instituto de Salud Carlos III FEDER (grant number 09/00629), FIS (PI10/00523 and PI10/01404), SEPAR 2010-870, Mutual Medica (DN040371) and Marató TV3 (040430). Funding information for this article has been deposited with the Open Funder Registry.

Conflict of interest: Disclosures can be found alongside this article at openres.ersjournals.com

The content of this work is @ the authors or their employers. Design and branding are @ERS 2016. This article is open access and distributed under the terms of the Creative Commons Attribution Non-Commercial Licence 4.0. 


\section{Introduction}

Chronic obstructive pulmonary disease (COPD) is associated with an enhanced pulmonary and systemic inflammatory response $[1,2]$ that bursts during exacerbations of the disease (ECOPD) [3]. The molecular mechanisms regulating this response, however, have not been fully elucidated $[1,2]$.

The regulation of the inflammatory response is highly complex. The Nod-like receptor (NLR) family plays a pivotal role [4-7] and one of its members, the so-called NLRP3 (NLR containing a Pyrin domain 3) inflammasome, has been implicated in different animal models of lung inflammation [4, 5, 8-10]. In patients with COPD, the evidence for NLRP3 inflammasome activation is scarce and contradictory [11-13], and has never been investigated before during ECOPD. Accordingly, in this study we investigated the role of the NLRP3 inflammasome in COPD patients, both when clinically stable and during ECOPD, smokers with normal spirometry and healthy controls.

\section{Methods}

\section{Study design and ethics}

This is an observational, prospective and controlled study in human samples. The ethics committees of participating institutions approved the study and all participants signed their informed consent.

\section{Participants}

We studied lung tissue specimens $(n=68)$ provided by the Pulmonary Biobank of CIBERES [14], which were obtained from patients undergoing lung resectional surgery because of localised lung cancer $(n=54)$ or bilateral lung transplantation $(n=14) .38$ of them had COPD [2] $(n=26,71 \%$ on inhaled long-acting bronchodilators and corticosteroids), 15 were smokers (>10 pack-years) with normal spirometry and 15 were never-smokers (table 1). We also obtained plasma and spontaneous sputum samples in another 56 patients hospitalised because of ECOPD (V1, during the first $72 \mathrm{~h}$ of hospitalisation) and when clinically stable ( $\mathrm{n}=41), 2$ months after discharge (V2) (table 1), $\mathrm{n}=15$ patients were lost for the follow-up or not able to produce sputum at V2. Six individuals $(10 \%)$ of the ECOPD subset received antibiotics prior to hospital admission.

\section{Measurements}

Lung function

Forced spirometry was measured in all participants according to international guidelines [15]. Reference values correspond to a Mediterranean population [16].

\section{Lung mRNA expression}

Total RNA was extracted from RNA later preserved tissue using a Purelink RNA minikit (Invitrogen, Paisley, UK) and cDNA was synthesised with Transcriptor Reverse Transcriptase (Roche, Mannheim, Germany). The relative quantification of each gene was normalised to the expression levels of glyceraldehyde 3-phosphate dehydrogenase as housekeeping gene using the $\Delta \mathrm{C}_{\mathrm{t}}$ method [17], as described in the supplementary material.

\section{IL-1 $\beta, I L-18$ and caspase-1 protein levels in lung and sputum}

Total lung proteins were obtained as detailed in the supplementary methods. Levels of caspase-1 and IL-18 were measured by ELISA (R\&D Systems, Minneapolis, MN, USA and MBL International, Woburn, MA,

\section{TABLE 1 Demographic and clinical characteristics of the individuals}

$\begin{array}{lccccc} & \text { Nonsmokers } & \text { Smokers } & \text { COPD } & \text { ECOPD } & \text { Recovery } \\ \text { Subjects } & 15 & 15 & 38 & 56 & 41 \\ \text { Age years } & 66 \pm 1.4 & 60 \pm 2.0 & 62 \pm 1.2 & 68.9 \pm 1.2 & 68.1 \pm 1.3 \\ \text { BMI kg.m }{ }^{-2} & 27.1 \pm 0.8 & 24.6 \pm 1.4 & 26.2 \pm 0.8 & 26.5 \pm 0.8 & 26.3 \pm 0.8 \\ \text { Cumulative smoking exposure pack-years } & 0 & 52.8 \pm 5.7 & 61.3 \pm 4.7 & 72.9 \pm 4.8 & 72.7 \pm 5.3 \\ \text { Smoking status ex/current } & 0 & 0 / 15 & 26 / 12 & 21 / 35 & 19 / 29 \\ \text { FEV } \% \text { reference } & 94.3 \pm 5.2 & 82.4 \pm 3.6 & 42.4 \pm 2.9 & 44.4 \pm 2.8 & 44.4 \pm 2.8 \\ \text { FEV } 1 \text { /FVC \%" } & 76.4 \pm 1.7 & 83.0 \pm 0.6 & 44.1 \pm 2.4 & 46.7 \pm 2.0 & 47.1 \pm 2.3\end{array}$

Data are presented as $\mathrm{n}$ or mean \pm SEM, unless otherwise stated. COPD: chronic obstructive pulmonary disease; ECOPD: exacerbations of COPD; BMI: body mass index; FEV1: forced expiratory volume in $1 \mathrm{~s}$; FVC: forced vital capacity. ${ }^{\#}$ : FEV $1 \%$ reference and FEV $_{1} / F V C$ values for nonsmokers and smokers are pre-bronchodilator, and for COPD patients are post-bronchodilator. 
USA, respectively). IL-1 $\beta$ concentration was determined by ultrasensitive Luminex (MILLIPLEX MAG; EMD Millipore, Billerica, MA, USA).

\section{Assessment of inflammasome activation}

The presence of active caspase-1 and ASC oligomers were assessed by Western blot using the anti-caspase-1 sc-515 antibody (Santa Cruz Biotechnology, Dallas, TX, USA), which detects the fragments of the active caspase-1 (10 kDa, p10) and the inactive uncleaved caspase-1 precursor $(45 \mathrm{kDa}, \mathrm{p} 45)$, or the anti-ASC antibody AL177 (Adipogen, Liestal, Switzerland), which identifies ASC monomers (22 kDa) and oligomers when using native gels as detailed in the supplementary methods.

\section{Caspase-1 and ASC lung tissue distribution}

The distribution of total caspase-1 was determined by immunohistochemistry using the anti-caspase-1 antibody AHP963 (AbD Serotec, Kidlington, UK). ASC distribution and ASC speck presence was assessed by immunofluorescence with an anti-ASC antibody (AL177) as detailed in the supplementary methods.

\section{Inflammation markers in ECOPD blood}

White blood cell counts were determined by standard automated methodology, whereas ultra-sensitive C reactive protein (CRP) in plasma was quantified by a turbidimetric test (Bayer Diagnostics, Munich, Germany).

Microbiology

Spontaneous sputum samples were cultured for Haemophilus influenzae, Streptococcus pneumoniae, Streptococcus viridans, Pseudomonas aeruginosa and Citrobacter ssp. Respiratory RNA viruses were detected by PCR (CLART ${ }^{\oplus}$ PneumoVir; Genomica, Madrid, Spain). An ECOPD episode was considered of infectious origin when a positive bacterial culture and/or a positive viral PCR were identified [18]. It was considered noninfectious when both were negative.

\section{Statistical analysis}

Results are presented as mean士sEm. The Kruskal-Wallis test, followed by post hoc Mann-Whitney contrasts if needed, was used to compare continuous variables between groups (COPD versus smokers versus nonsmokers). Results during ECOPD and recovery were compared using the Wilcoxon matched-pairs test. A p-value $<0.05$ (two-tailed) was considered statistically significant.

\section{Results}

Table 1 summarises the main characteristics of the patients included in the study.

\section{NLRP3 expression is increased in stable COPD lungs and correlates with airflow obstruction} NLRP3 mRNA levels were significantly higher in the lungs of stable COPD patients versus nonsmokers and smokers with normal spirometry (figure 1a). This increase was independent of the smoking status of the patient (current versus former) (supplementary figure S1) and was related to the severity of airflow limitation present (figure 1b). Furthermore, NLRP3 expression levels in COPD positively correlated with different inflammatory mediators that are both canonical and noncanonical to the NLRP3 inflammasome, including IL-1 $\beta$ and IL-18 (figure $1 \mathrm{c}$ and $\mathrm{d}$ ), and IL-1 $\alpha$, IL-8, neutrophil elastase, CCL20 and IL-2 (supplementary figure S2).

\section{IL-1ß, but not IL-18, expression correlates with airflow limitation in stable COPD}

IL-1 $\beta$ transcripts tended to increase in smokers and, even further, in stable COPD patients versus nonsmokers, (figure 2a), but differences just failed to reach statistical significance (Kruskal-Wallis test, $\mathrm{p}=0.06$ ). There were no differences in IL-18 transcripts between groups, confirming previous reports of a noninducible expression of IL-18. IL-1 $\beta$, but not IL-18, lung transcripts were related to the severity of airflow limitation in COPD patients (figure $2 \mathrm{~b}$ and data not shown).

Although both IL-1 $\beta$ and IL-18 protein levels in lung tissue were increased in COPD versus control nonsmokers, these differences failed to reach statistical significance (figure 2c). Interestingly, these cytokines were significantly higher in smokers when compared with healthy individuals (figure 2c). No significant differences were observed between COPD patients according to their smoking habits (current versus former smokers) (supplementary figure S1). Similarly, no differences between patients treated with or without inhaled corticosteroids (data not shown).

Stable COPD is not associated with inflammasome or caspase-1 activation in lung tissue Lung mRNA and protein levels for caspase-1 measured by quantitative PCR and ELISA, respectively, were similar between nonsmokers, smokers and COPD (figure 3a). Furthermore, Western blot analysis of 

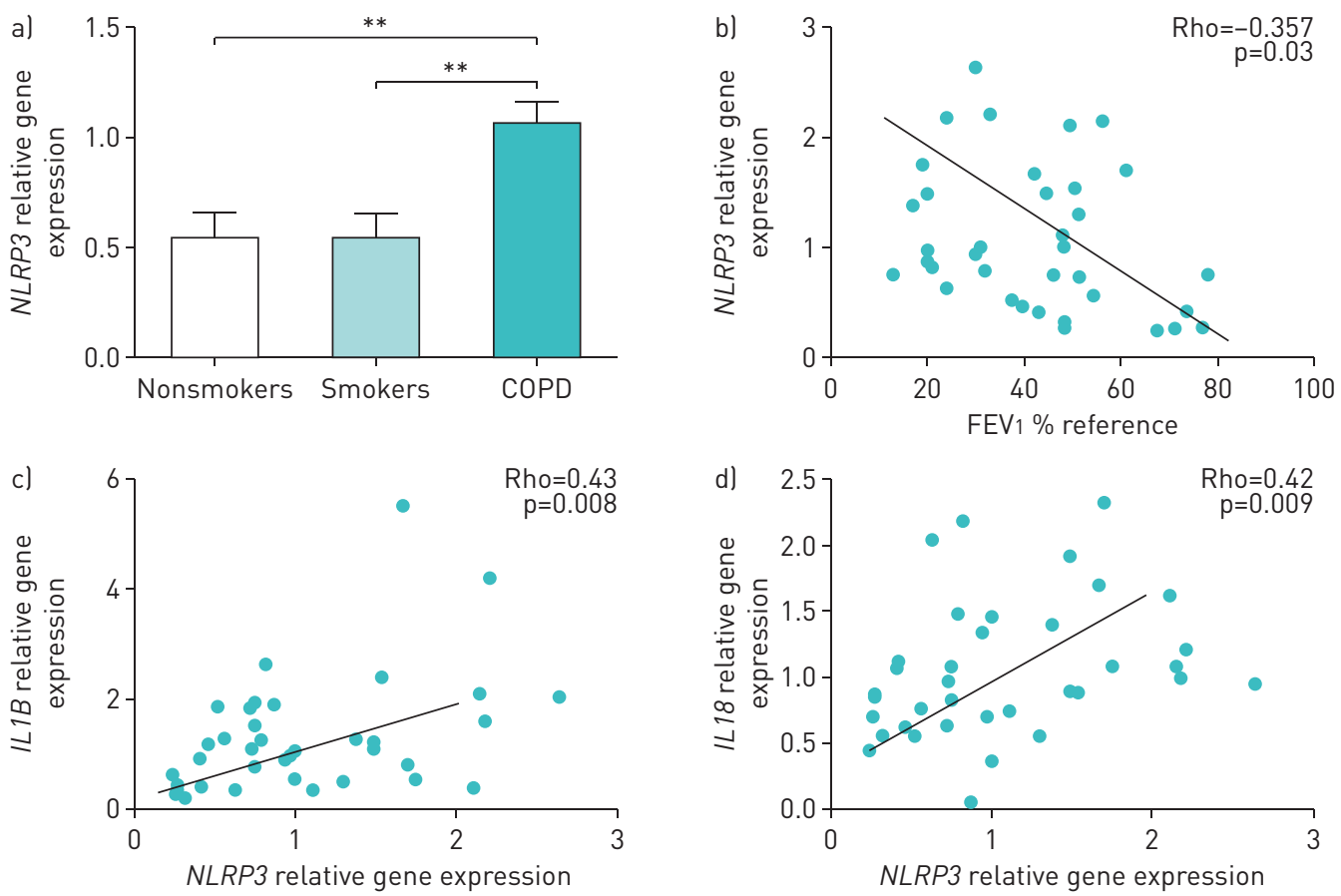

FIGURE 1 NLRP3 expression in lung tissue. a) NLRP3 relative transcript levels in lung tissues of nonsmokers, smokers or chronic obstructive pulmonary disease (COPD) patients (relative quantification of mRNA levels relative to glyceraldehyde 3-phosphate dehydrogenase). b) Spearman correlation of NLRP3 mRNA expression levels in lungs of patients with stable COPD with forced expiratory volume in $1 \mathrm{~s}$ (FEV $1 \%$ reference). c, d) Spearman correlation of NLRP3 expression with the levels of c) ILIB and d) IL18 transcripts. a) Data is presented as mean \pm SEM of $n=14$ nonsmokers, $n=15$ smokers and $n=38$ COPD individuals; $b-d$ ) each point represents an individual patient. ${ }^{* *}: p<0.005$.

caspase- 1 revealed that it was mainly in the inactive pro-form (p45) (figure $3 \mathrm{~b}$ ). The band corresponding to the small active subunit of caspase-1 (p10) was only detected in $29 \%$ of the COPD patients, $20 \%$ of nonsmokers and $27 \%$ of smokers (figure $3 \mathrm{~b}$ ). Finally, in stable COPD patients, caspase-1 expression was detected by immunohistochemistry in lung epithelial cells and inflammatory cells (mostly macrophages and lymphocytes) (figure $3 \mathrm{~d}$ and $\mathrm{f}$ ). However, a similar expression pattern for caspase-1 was observed in the lungs of control nonsmokers when compared with stable COPD patients (figure 3c-f). Similarly, ASC appeared mainly in the monomeric state in COPD patients and in nonsmokers (figure $3 \mathrm{~g}$ ); weak oligomers for ASC were also detected in both COPD patients and controls (figure 3g). ASC oligomeric species were reversed when samples were treated with a reducing agent prior to resolving by SDS-PAGE (figure $3 \mathrm{~g}$ ). Finally, the pattern of ASC staining in lung tissue (figure $3 \mathrm{~h}-\mathrm{j}$ ) resembles that described for caspase-1 and was similar in all groups of patients examined. ASC specks (oligomers) were detected by immunofluorescence in some macrophages (figure $3 \mathrm{~h}-\mathrm{j}$ ), but similar to caspase-1, the majority of ASC immunostaining was mainly detected as uniform staining through the cell cytosol, probably corresponding to the inactive monomeric form of ASC (figure $3 \mathrm{~h}-\mathrm{j}$ ).

\section{Infectious ECOPD episodes are associated with inflammasome activation}

During ECOPD we observed leukocytosis, neutrophilia, lymphopenia and elevated C-reactive protein values (table 2). In the entire population of COPD patients studied, the concentrations of released caspase-1, IL-1 $\beta$ and IL-18 in sputum were similar during the ECOPD episode and at recovery (figure 4a).

Next, patients were stratified according to the nature of exacerbation. An ECOPD episode was considered infectious when a positive bacterial culture and/or a positive viral PCR were identified [18, 19]. In infectious ECOPD, $53 \%$ of the cases were of viral origin, $41 \%$ were bacterial and only $6 \%$ had both causes. The ECOPD was considered noninfectious when both viral PCR and bacterial culture were negative. We observed that the sputum concentrations of caspase-1, IL-1 $\beta$ and IL-18 were significantly higher when infection was present (figure 4b).

During ECOPD we detected oligomeric ASC in sputum, a hallmark of inflammasome activation (figure 4c), indicating that extracellular oligomers of ASC are present during ECOPD. As a methodological control, we show that these ASC oligomers disappeared when samples were reduced prior to analysis (figure 4c). The data support recent reports describing an extracellular function of inflammasome particles [20, 21]. 

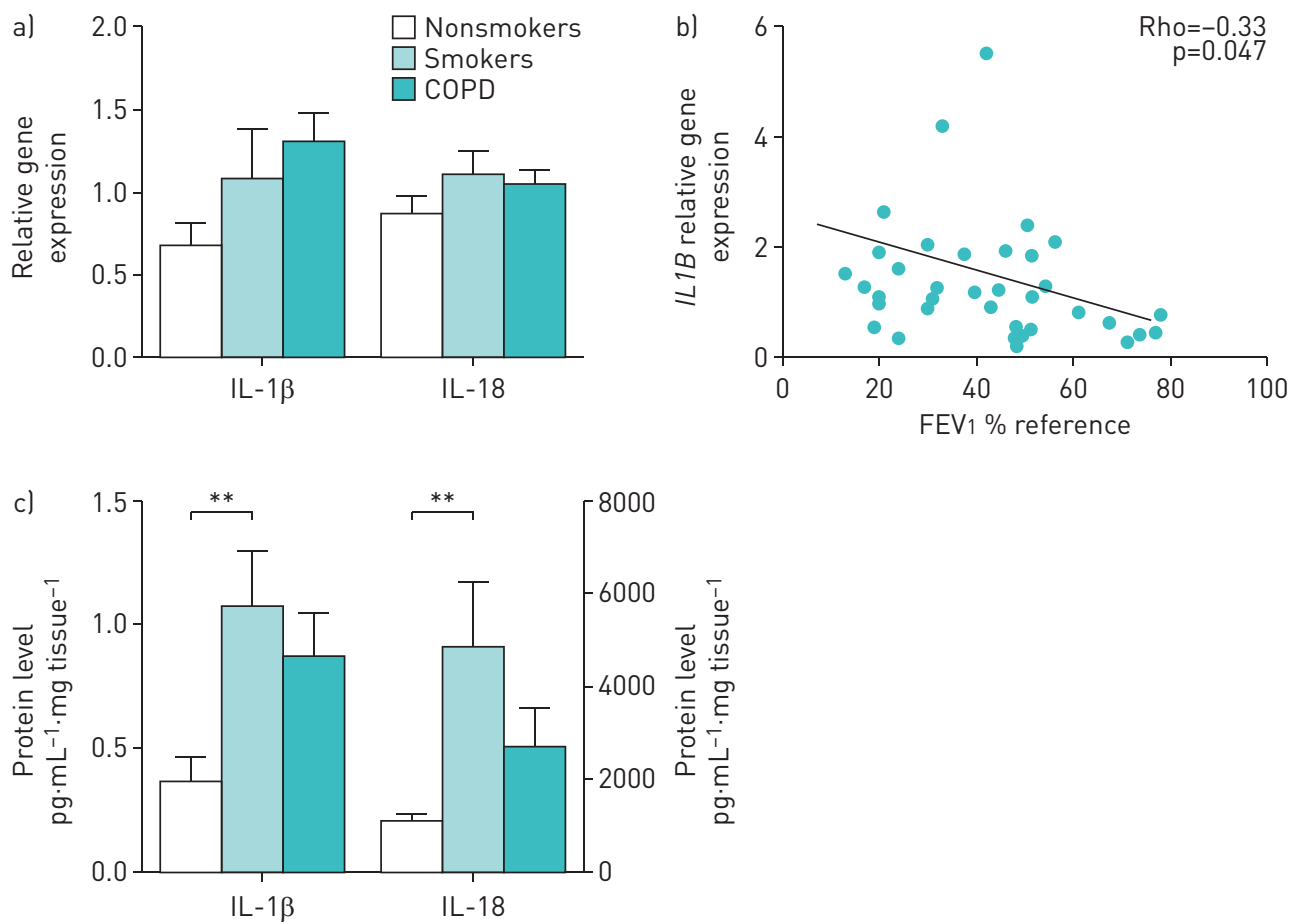

FIGURE 2 Interleukin (IL)-1 $\beta$ and IL-18 in stable chronic obstructive pulmonary disease (COPD) patients. a) $\mathrm{IL}-1 \beta$ and IL-18 relative transcript levels in lung tissues of nonsmokers, smokers or COPD patients (relative quantification of mRNA levels relative to glyceraldehyde 3-phosphate dehydrogenase). b) Spearman correlation of IL-1 $\beta$ mRNA expression levels in lungs of patients with stable COPD with forced expiratory volume in $1 \mathrm{~s}$ (FEV $1 \%$ reference). $\mathrm{c}$ ) $\mathrm{IL}-1 \beta$ and $\mathrm{IL}-18$ protein levels measured by Luminex and ELISA, respectively, in lung tissue. a, c) Data is presented as mean \pm SEM of $n=14$ nonsmokers, $n=15$ smokers and $n=38$ COPD individuals; b) each point represents an individual patient. ${ }^{* *}: p<0.01$.

\section{Discussion}

This study shows that: 1) NLRP3 and IL-1 $\beta$ mRNA are upregulated in lung tissue of stable COPD, but both caspase- 1 and ASC are found mostly in inactive form, suggesting that the NLRP3 inflammasome is primed, but not activated, in stable COPD, and 2) during infectious ECOPD, caspase-1, oligomeric ASC and associated cytokines (IL-1 $\beta$, IL-18) are upregulated in sputum, indicating that inflammasome activation occurs under these circumstances.

\section{Previous studies}

Over the last few years, knowledge about the inflammasome function in different inflammatory conditions has increased exponentially, mostly as a result of knockout mice and in vitro cell assays [20, 22, 23]. For instance, the pulmonary inflammatory response is blunted in NLRP3 knockout mice [4, 5, 9]. In patients with COPD, the evidence for inflammasome activation is scarce and contradictory. While some studies reported increased levels of IL-1 $[11,12]$, others failed to find NLRP3, or increase in IL-1 $\beta$ and IL-18 protein in stable COPD patients [13]. Most of them were, however, of limited size and, contrary to our study, did not assess whether the inflammasome is a primer or active.

\section{Interpretation of findings}

The inflammasomes form a family of multimeric intracellular protein complexes that most often include a NLR as a sensor molecule and, in some cases, the adaptor protein apoptosis-associated speck-like protein containing a caspase recruitment domain (ASC) and pro-caspase-1 [24, 25]. The best-characterised inflammasome is that formed by NLRP3, which is activated in response to different pathogens, particles and danger signals $[24,25]$. Full activation of the NLRP3 inflammasome requires two sequential biological steps. The first (priming step) increases pro-IL-1 $\beta$ and NLRP3 transcription, and the second (activation step) induces inflammasome assembly, activation of caspase-1 and processing, and release of the pro-inflammatory cytokines IL-1 $\beta$ and IL-18 $[24,25]$. Priming is related to the presence of inflammation, whereas activation occurs only after exposure to specific danger signals [24, 25]. The activation involves the oligomerization of NLRP3 with ASC, in a large fibre-like complex called "speck", which can be detected by immunofluorescence or by ASC cross-linking in Western blots [26-28]. NLRP3 and IL-1 $\beta$ 


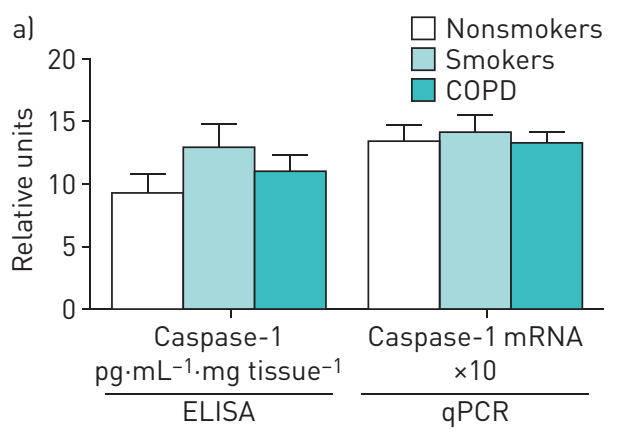

b)
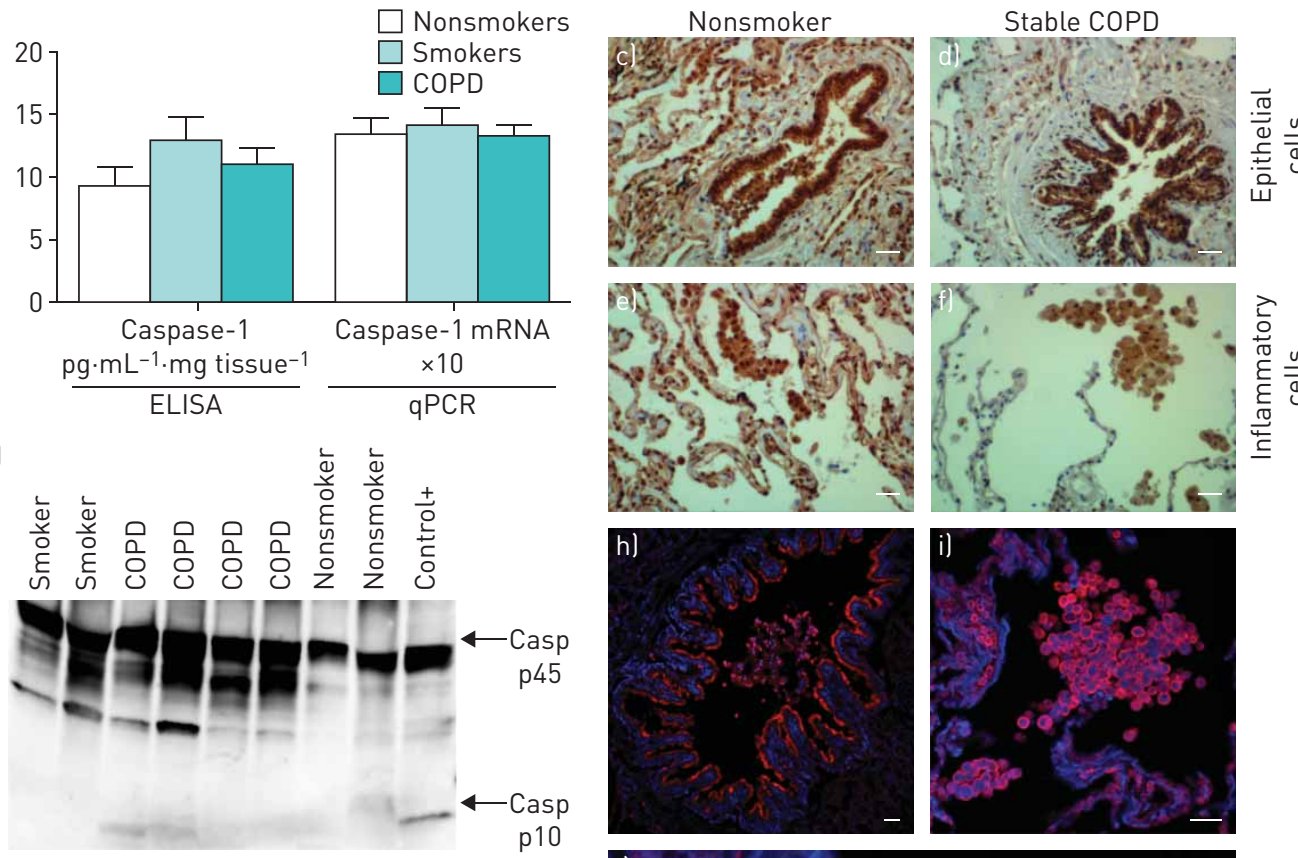

g)
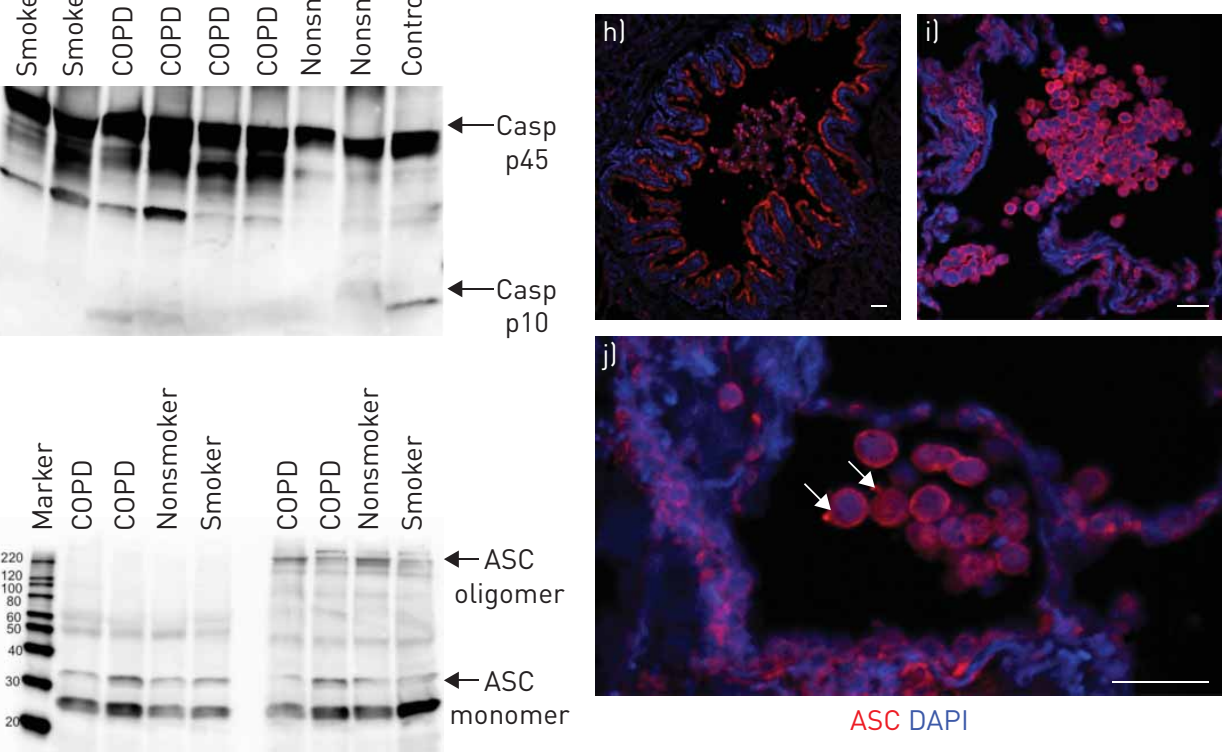

ASC DAPI

Reduced

Nonreduced

FIGURE 3 Inflammasome is not activated in stable chronic obstructive pulmonary disease (COPD). a) Caspase-1 protein levels measured by ELISA and relative mRNA expression measured by quantitative PCR (qPCR) in lung tissue of stable COPD. Data is presented as mean \pm SEM of $n=14$ nonsmokers, $n=15$ smokers and $n=38$ COPD individuals. b) Representative Western blot analysis of caspase-1 (Casp); positive control (+) is a lysate of human peripheral blood mononuclear cells treated with the NLRP3 activator nigericin (10 $\mu \mathrm{M})$. c-f) Caspase-1-positive cells (brown) detected by immunohistochemistry in c, d) bronchial epithelial cells and alveolar epithelial cells and e, f) macrophages in a representative tissue sample from a nonsmoker and a COPD patient. The primary antibody used in immunohistochemistry detects both the p45 inactive precursor and the small subunit of active caspase-1 (p10). Scale bars: $100 \mu \mathrm{m}$. g) Western blot for ASC in representative lung samples from two COPD patients, a nonsmoker (NS) and a smoker (S), denoting the monomeric (22 kDa) and oligomeric (220 kDa) ASC. ASC oligomeric complexes were not detected when samples were run under reduced conditions. $h-j)$ ASC immunofluorescence staining (red) and nuclei (blue) in lung tissue of a representative COPD patient: h) alveolar epithelial cells and macrophages, i) bronchial epithelial cells, and j) macrophages. Arrows in j) denote macrophages with detectable ASC speck. DAPI: 4',6-diamidino-2-phenylindole. Scale bars: $36 \mu \mathrm{m}$.

mRNA levels are generally considered markers of inflammasome priming, whereas NLRP3 and ASC oligomerization and presence of active caspase-1 are readouts of inflammasome activation [20, 21, 29-31].

In line with previous studies reporting an association of IL- $1 \beta$ with COPD $[4,5,8]$, we found increased NLRP3 transcripts in the lungs of stable COPD patients. In addition, we also found that NLRP3 mRNA expression correlated positively with the severity of airflow limitation, and with mRNA expression for IL-1 $\alpha$, IL-18, IL-1 $\beta$, IL- 8 and neutrophil elastase. However, we could not observe any direct indicator of inflammasome formation and activation during stable COPD. These results are in keeping with those reported by Di Stefano et al. [13], but in contrast to those by Ецтом et al. [32], who reported increased caspase-1 activity in lung tissue of patients with emphysema. Although we observed increased protein levels of IL-1 $\beta$ and IL-18 in current smokers with normal spirometry, current smoking did not influence the levels of these proteins in patients with COPD. Accordingly, these findings suggest that the production of IL- $1 \beta$ and IL-18 is driven by a different mechanism in each situation. 


\begin{tabular}{|c|c|c|c|}
\hline & ECOPD & Recovery & p-value \\
\hline Subjects & 56 & 41 & \\
\hline Leukocytes $\times 10^{3} \mu \mathrm{L}^{-1}$ & $11.59 \pm 0.47$ & $8.67 \pm 0.3$ & $<0.0001$ \\
\hline Neutrophils $\times 10^{3} \mu \mathrm{L}^{-1}$ & $85.4 \pm 1.3$ & $62.6 \pm 1.6$ & $<0.0001$ \\
\hline Lymphocytes $\times 10^{3} \mu \mathrm{L}^{-1}$ & $9.3 \pm 0.6$ & $24.35 \pm 1.0$ & $<0.0001$ \\
\hline C-reactive protein $\mathrm{mg} \cdot \mathrm{dL}^{-1}$ & $6.5 \pm 0.9$ & $1.1 \pm 0.3$ & $<0.0001$ \\
\hline
\end{tabular}

However, we also found, for the first time to our knowledge, that the inflammasome becomes activated in human lungs during infectious ECOPD. This was evidenced by the increased sputum concentrations of caspase-1, IL-1 $\beta$ and IL-18 observed in infectious ECOPD (as compared with noninfectious exacerbations). The fact that we found these cytokines in sputum supernatant indicates that these proteins have actually been released from the cells, suggesting that they correspond to their active forms [30, 31]. In keeping with this interpretation is the fact that we also detected oligomers of ASC in sputum during infectious ECOPD, supporting recent studies indicating that ASC oligomeric particles are released from activated macrophages and contribute to perpetuate inflammation [20,21]. The fact that cell-free ASC oligomeric particles have been observed in the bronchoalveolar lavage of a small series of COPD patients further supports these observations [21]. Finally, our results are also in agreement with those reported by BAFADHEL et al. [19], which showed different patterns of inflammatory response during ECOPD and reported that the biomarker
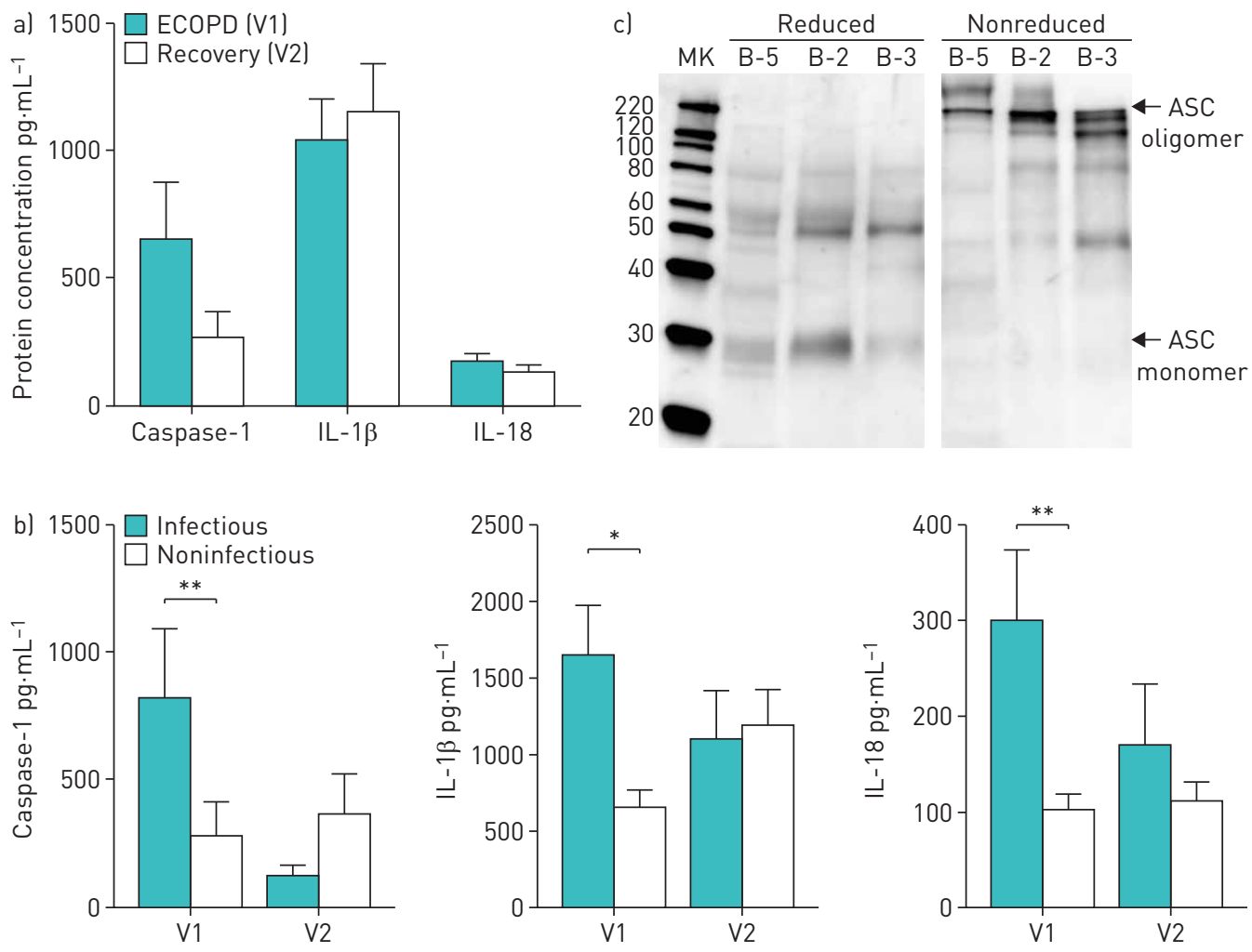

FIGURE 4 NLRP3 inflammasome is activated during infectious exacerbations of chronic obstructive pulmonary disease (ECOPD). a) Protein concentration of caspase-1, interleukin (IL)-1 $\beta$ and IL-18 in sputum of COPD patients studied during an ECOPD (V1) and at recovery (V2). Data are presented as mean \pm SEM of $n=41$ (V1) and $\mathrm{n}=41$ (V2). b) Sputum concentrations of caspase-1, IL-1 $\beta$ and IL-18 in COPD patients studied during an episode of ECOPD of infectious or noninfectious origin. Data are presented as mean \pm SEM of $n=17$ infectious V1 (during ECOPD), $n=14$ infectious V2 (recovery), $n=30$ noninfectious V1 and $n=21$ noninfectious V2 individuals. *: $p<0.05$; ${ }^{* *}: p<0.01$. c) Representative Western blot for ASC sputum samples from ECOPD patients, denoting oligomeric ASC complexes, a hallmark of inflammasome activation. ASC oligomeric complexes were not detected when samples were run under reduced conditions. MK: marker. 
that best identified ECOPD of bacterial origin was sputum IL-1 $\beta$ concentration. Whether or not this inflammasome response in infectious ECOPD can be protective or detrimental to the host requires further research. The results of our study show that the inflammasome is involved in the inflammatory burst of infectious ECOPD. It is plausible that controlling the primed status of the inflammasome in stable COPD might result in a decrease in the inflammasome-induced inflammation observed in infectious ECOPD.

\section{Strengths and limitations}

This is the largest study investigating the participation of the NLRP3 in lung tissue samples of COPD patients (and appropriate controls) and the first to assess its potential involvement during episodes of ECOPD. It has, however, some limitations. Due to clinical constrains, we were unable to obtain all measurements in all patients and lung tissue samples were not accessible during ECOPD. ECOPD blood and sputum samples were collected during the first $72 \mathrm{~h}$ of hospitalisation, but we did not have information at discharge and during recovery. 98\% of ECOPD patients were on inhaled corticosteroids previously, so that differences between infectious noninfectious groups could not be attributed to different treatments. Finally our definition of infectious ECOPD (bacterial culture and/or viral PCR), although previously used in similar studies [19], may potentially lead to over/underdiagnosis.

\section{Conclusions}

Our results indicate that NLRP3 is primed in the lungs of patients with clinically stable COPD and that infectious ECOPD constitutes a robust second signal that triggers inflammasome activation.

\section{Acknowledgements}

The authors thank the participating patients for their willingness to contribute to biomedical research, Ms Gemma Sunyer (IDIBAPS, Barcelona, Spain) for her excellent technical support during the study, and Dr Cristina Villena (CIBERES Pulmonary Biobank, Barcelona, Spain) and Drs Joan Albert Barbera Barberá and Victor I. Peinado (IDIBAPS, Barcelona, Spain) for providing the tissue samples used in the study, as well as all clinical investigators of the ECOS (Exacerbations of COPD in Spain) study.

\section{References}

1 Cosio MG, Saetta M, Agusti A. Immunologic aspects of chronic obstructive pulmonary disease. $N$ Engl J Med 2009; 360: 2445-2454.

2 Vestbo J, Hurd SS, Agusti AG, et al. Global strategy for the diagnosis, management, and prevention of chronic obstructive pulmonary disease: GOLD executive summary. Am J Respir Crit Care Med 2013; 187: 347-365.

3 Perera WR, Hurst JR, Wilkinson TM, et al. Inflammatory changes, recovery and recurrence at COPD exacerbation. Eur Respir J 2007; 29: 527-534.

4 Yazdi AS, Guarda G, Riteau N, et al. Nanoparticles activate the NLR pyrin domain containing 3 (Nlrp3) inflammasome and cause pulmonary inflammation through release of IL-1alpha and IL-1beta. Proc Natl Acad Sci USA 2010; 107: 19449-19454.

5 Gasse P, Mary C, Guenon I, et al. IL-1R1/MyD88 signaling and the inflammasome are essential in pulmonary inflammation and fibrosis in mice. J Clin Invest 2007; 117: 3786-3799.

6 Lucattelli M, Cicko S, Muller T, et al. P2X7 receptor signaling in the pathogenesis of smoke-induced lung inflammation and emphysema. Am J Respir Cell Mol Biol 2011; 44: 423-429.

7 Gasse P, Riteau N, Charron S, et al. Uric acid is a danger signal activating NALP3 inflammasome in lung injury inflammation and fibrosis. Am J Respir Crit Care Med 2009; 179: 903-913.

8 Riteau N, Gasse P, Fauconnier L, et al. Extracellular ATP is a danger signal activating P2X7 receptor in lung inflammation and fibrosis. Am J Respir Crit Care Med 2010; 182: 774-783.

9 Cassel SL, Eisenbarth SC, Iyer SS, et al. The Nalp3 inflammasome is essential for the development of silicosis Proc Natl Acad Sci USA 2008; 105: 9035-9040.

10 Lommatzsch M, Cicko S, Muller T, et al. Extracellular adenosine triphosphate and chronic obstructive pulmonary disease. Am J Respir Crit Care Med 2010; 181: 928-934.

11 Chung KF. Cytokines in chronic obstructive pulmonary disease. Eur Respir J Suppl 2001; 34: 50s-59s

12 Gessner C, Scheibe R, Wotzel M, et al. Exhaled breath condensate cytokine patterns in chronic obstructive pulmonary disease. Respir Med 2005; 99: 1229-1240.

13 Di Stefano A, Caramori G, Barczyk A, et al. Innate immunity but not NLRP3 inflammasome activation correlates with severity of stable COPD. Thorax 2014; 69: 516-524.

14 Villena C, Pozo F, Barbera JA, et al. The CIBERES Pulmonary Biobank Consortium: an opportunity for cooperative international respiratory research. Eur Respir J 2011; 37: 204-206.

15 American Thoracic Society. Standardization of Spirometry, 1994 Update. Am J Respir Crit Care Med 1995; 152: 1107-1136.

16 Roca J, Sanchis J, Agusti-Vidal A, et al. Spirometric reference values from a Mediterranean population. Bull Eur Physiopathol Respir 1986; 22: 217-224.

17 Livak KJ, Schmittgen TD. Analysis of relative gene expression data using real-time quantitative PCR and the 2(-Delta Delta C(T)) method. Methods 2001; 25: 402-408.

18 Rodriguez-Roisin R. Toward a consensus definition for COPD exacerbations. Chest 2000; 117: 5 Suppl. 2, 398S-401S.

19 Bafadhel M, McKenna S, Terry S, et al. Acute exacerbations of chronic obstructive pulmonary disease: identification of biologic clusters and their biomarkers. Am J Respir Crit Care Med 2011; 184: 662-671.

20 Baroja-Mazo A, Martin-Sanchez F, Gomez AI, et al. The NLRP3 inflammasome is released as a particulate danger signal that amplifies the inflammatory response. Nat Immunol 2014; 15: 738-748. 
21 Franklin BS, Bossaller L, De Nardo D, et al. The adaptor ASC has extracellular and 'prionoid' activities that propagate inflammation. Nat Immunol 2014; 15: 727-737.

22 Franchi L, Nunez G. The Nlrp3 inflammasome is critical for aluminium hydroxide-mediated IL-1beta secretion but dispensable for adjuvant activity. Eur J Immunol 2008; 38: 2085-2089.

23 Franchi L, Eigenbrod T, Nunez G. Cutting edge: TNF-alpha mediates sensitization to ATP and silica via the NLRP3 inflammasome in the absence of microbial stimulation. J Immunol 2009; 183: 792-796.

24 Schroder K, Tschopp J. The inflammasomes. Cell 2010; 140: 821-832.

25 Latz E, Xiao TS, Stutz A. Activation and regulation of the inflammasomes. Nat Rev Immunol 2013; 13: 397-411.

26 Stutz A, Horvath GL, Monks BG, et al. ASC speck formation as a readout for inflammasome activation. Methods Mol Biol 2013; 1040: 91-101.

$27 \mathrm{Lu} \mathrm{A}$, Magupalli VG, Ruan J, et al. Unified polymerization mechanism for the assembly of ASC-dependent inflammasomes. Cell 2014; 156: 1193-1206.

28 Cai X, Chen $\mathrm{J}, \mathrm{Xu} \mathrm{H}$, et al. Prion-like polymerization underlies signal transduction in antiviral immune defense and inflammasome activation. Cell 2014; 156: 1207-1222.

29 Lee JK, Kim SH, Lewis EC, et al. Differences in signaling pathways by IL-1beta and IL-18. Proc Natl Acad Sci USA 2004; 101: 8815-8820.

30 Sarkar A, Mitra S, Mehta S, et al. Monocyte derived microvesicles deliver a cell death message via encapsulated caspase-1. PLoS One 2009; 4: e7140.

31 Laliberte RE, Eggler J, Gabel CA. ATP treatment of human monocytes promotes caspase-1 maturation and externalization. J Biol Chem 1999; 274: 36944-36951.

32 Eltom S, Stevenson CS, Rastrick J, et al. P2X7 receptor and caspase 1 activation are central to airway inflammation observed after exposure to tobacco smoke. PLoS One 2011; 6: e24097. 